\title{
Segregation analysis
}

National Cancer Institute ( $\mathrm{NCl})$

\section{Source}

National Cancer Institute (NCI). Segregation analysis.

The process of fitting formal genetic models to data on expressed disease

characteristics (phenotype) in biological family members in order to determine the most

likely mode of inheritance for the trait or disease under study. 